TITLE:

\title{
Fiber tract associated with autistic traits in healthy adults.
}

\section{AUTHOR(S):}

Hirose, Kimito; Miyata, Jun; Sugihara, Genichi; Kubota, Manabu; Sasamoto, Akihiko; Aso, Toshihiko; Fukuyama, Hidenao; Murai, Toshiya; Takahashi, Hidehiko

\section{CITATION:}

Hirose, Kimito ... [et al]. Fiber tract associated with autistic traits in healthy adults.. Journal of psychiatric research 2014, 59: 117-124

\section{ISSUE DATE:}

2014-12

URL:

http://hdl.handle.net/2433/191261

\section{RIGHT:}

(C) 2014 Elsevier Ltd.; This is not the published version. Please cite only the published version.; この論文は出版社版でありません。引用の際に は出版社版をご確認ご利用ください。 


\section{Fiber tract associated with autistic traits in healthy adults}

3 Kimito Hirose, M.D. ${ }^{a}$, Jun Miyata, M.D., Ph.D. ${ }^{a}$, Genichi Sugihara M.D., Ph.D. ${ }^{\text {a }}$, Manabu Kubota,

4 M.D., Ph.D. ${ }^{a}$, Akihiko Sasamoto M.D. ${ }^{\mathrm{a}}$, Toshihiko Aso, M.D., Ph.D. ${ }^{\mathrm{b}}$, Hidenao Fukuyama, M.D., Ph.D. ${ }^{\mathrm{b}}$,

5 Toshiya Murai, M.D., Ph.D. ${ }^{\mathrm{a}}$ and Hidehiko Takahashi, M.D., Ph.D. ${ }^{\mathrm{a}}$,

6

7 a Department of Psychiatry, Graduate School of Medicine, Kyoto University, 54

8 Shogoin-Kawaharacho, Sakyo-ku, Kyoto 606-8507, Japan

$9 \quad$ bHuman Brain Research Center, Graduate School of Medicine, Kyoto University, 54

10 Shogoin-Kawaharacho, Sakyo-ku, Kyoto 606-8507, Japan

11

12

Please address correspondence to:

13 Hidehiko Takahashi, M.D., Ph.D.

14

Department of Psychiatry, Graduate School of Medicine, Kyoto University 54

15

Shogoin-Kawaharacho, Sakyo-ku, Kyoto 606-8507, Japan

16

Tel: +81-75-751-4947, Fax: +81-75-751-3246

17

E-mail: hidehiko@kuhp.kyoto-u.ac.jp

18 


\section{Abstract}

Autism Spectrum Disorder (ASD) is a neurodevelopmental disorder with impairment of social

3 communication and restricted and repetitive behaviors. Reduced fractional anisotropy (FA), a measure of

4 white matter integrity, in the posterior superior temporal sulcus (pSTS) is related to ASD. However, there

5 are several major fibers in pSTS, and it is unknown which of them is associated with ASD. We

6 investigated FA in correlation with autistic traits assessed by autism spectrum quotient (AQ) in 91 healthy

7 adults using tract-based spatial statistics (TBSS). Then, of the fibers in pSTS, we identified the one in

8 which FA was linked to the AQ score using tractography. TBSS revealed that AQ was correlated with FA

9 of white matter in several regions such as the frontal lobe, parietal lobe, occipital lobe and temporal lobe

10 including pSTS. With further analysis using tractography, we confirmed that FA alteration in pSTS was

11 located on the inferior fronto-occipital fasciculus (IFOF). IFOF has a critical role in processing

12 socio-emotional information. Our findings suggest that of the fibers in pSTS, IFOF is a key fiber that

13 links to autistic traits in healthy adults.

14

\section{Keywords}

16 Autism, tract-based spatial statistics, tractography, posterior superior temporal sulcus, inferior

17 fronto-occipital fasciculus 


\section{Introduction}

Autism Spectrum Disorder (ASD) was proposed as a developmental disorder with a triad of

3 impairments in social interaction, communication, and imagination (Wing, 1988, Wing, 1997), and it was

4 defined in the Diagnostic and Statistical Manual of Mental Disorders fifth edition (DSM-5) as

5 neurodevelopmental disorder with impairment of social communication and restricted and repetitive

6 behaviors (American Psychiatric Association, 2013). ASD was previously considered to have aberrant

7 brain function in specific regions such as the cerebral cortex, cerebellum, amygdala, basal ganglia, and

8 mesolimbic system (Allely et al., 2014, Cauda et al., 2011, Courchesne, 1997, Stanfield et al., 2008, Via

9 et al., 2011). Recently, ASD has been increasingly considered a disorder of impaired brain networks (Just

10 et al., 2012, Kana et al., 2011, Vissers et al., 2012), in which white matter abnormalities can be the neural

11 underpinnings. Previous diffusion tensor imaging (DTI) studies in people with ASD repeatedly reported

12 the reduction of fractional anisotropy (FA), which indicates impaired white matter integrity (Walker et al.,

13 2012). A recent study has examined the developmental trajectory of white matter in 6- to 24-month

14 infants with high risk of ASD, showing rapid increase and subsequent stoppage of FA of several fiber

15 tracts in very young infants with ASD and supporting the consistent findings of reduced FA in individuals

16 with ASD (Wolff et al., 2012). Brain alterations in individuals in ASD are also evident in high autistic

17 traits in healthy people (Nummenmaa et al., 2012, Wallace et al., 2012), which is in line with the notion

18 that ASD forms a continuum from autism to healthy population (Baron-Cohen et al., 2001a, Constantino

19 and Todd, 2003, Robinson et al., 2011). These findings suggest that there is reduced white matter integrity 
1 in the brain of people with ASD and that such alterations can be related to autistic traits in healthy people.

The posterior superior temporal sulcus (pSTS), which has many inputs and outputs of white matter

3 fibers (Lahnakoski et al., 2012), has been repeatedly reported to play a key role in the pathophysiology of

4 ASD (Pelphrey et al., 2011, Zilbovicius et al., 2006). In addition, growing evidence has indicated that

5 there is reduced FA of white matter around pSTS of people with ASD. Reduced FA in the white matter

6 region adjacent to pSTS has been reported by several DTI studies using voxel-based morphometry

7 (VBM) (Barnea-Goraly et al., 2004, Bloemen et al., 2010, Groen et al., 2011), a widely used automated

8 imaging-analysis method for exploring regional brain alterations. Recently, some studies used tract-based

9 spatial statistics (TBSS), which is a voxelwise analysis method for DTI and is robust for registration

10 errors inherent in VBM (Smith et al., 2006), to show a reduction of FA in the pSTS region in children and

11 adolescent with ASD (Barnea-Goraly et al., 2010, Jou et al., 2011b, Shukla et al., 2011a). However, it is

12 difficult from these voxelwise techniques to know on which fiber tracts these FA changes are located,

13 because several white matter tracts run adjacent to pSTS, including the arcuate fasciculus (AF), inferior

14 longitudinal fasciculus (ILF) and inferior fronto-occipital fasciculus (IFOF) (Catani and de Schotten,

15 2012).

16

How can we investigate the detailed location of FA change? Diffusion tractography reconstructs

17 each individual white matter fiber trajectory using directional information in DTI data. Integrating

18 tractography technique with voxelwise method is helpful for investigating on which fiber tracts FA is

19 reduced in the pSTS-adjacent white matter region. So far, two studies have employed this strategy. Kumar 
1 et al found reduced FA around the STS region in TBSS in young children with ASD and confirmed the

2 reduction of FA for several fibers using tractography, although their findings were not significant after

3 correcting for IQ (Kumar et al., 2010). Using VBM, Jou et al showed clusters of FA reduction in the pSTS

4 region in children with ASD, and then revealed by tractography that clusters can be either on IFOF or ILF

5 (Jou et al., 2011a). However, it still remains unclear which fibers in pSTS (e.g., ILF and IFOF) have FA

6 alterations in people with ASD.

7 In the current study, we aimed to identify the anatomy of autistic trait-related FA alterations in the

8 pSTS region with a method using TBSS and subsequent tractography. We used the autism spectrum

9 quotient (AQ), a well-validated self-report questionnaire of autistic tendency (Baron-Cohen et al., 2001b,

10 Wakabayashi et al., 2006), for a large sample of healthy adults and investigated regions where FA values

11 would be associated with autistic traits using TBSS. We then performed tractography and examined in

12 which fiber tract in pSTS such autistic-trait-related FA alterations were located.

\section{Materials and Methods}

16 Participants

17 Ninety-seven healthy adults participated in this study (mean age 28 years; age range, 19-55 years;

1847 females; 5 left-handed subjects). Autism spectrum traits were measured with AQ for all participants.

19 To assess the intelligence quotient (IQ), vocabulary task and block design of the Wechsler adult 
1 intelligence scale revised (WAIS-R) were carried out. The structured clinical interview for DSM-IV axis I

2 disorders (SCID) was performed by trained psychiatrists to check for current or past history of psychiatric

3 disorders. Two participants were excluded due to a history of depression and possible onset of

4 obsessive-compulsive disorder. Subjects underwent brain MRI according to the protocol described below.

5 MRI could not be performed for 1 subject due to cosmetics, and DTI images could not be used for

6 analysis of 3 subjects due to head motion. Finally, 91 participants were included in the analyses. The

7 research protocol was approved by the institutional review board and the ethics committee of Kyoto

8 University Graduate School of Medicine. Written informed consent was obtained from all participants at

9 the time of their visit.

11 Autism spectrum quotient (AQ)

We adopted the Japanese version of the AQ scale (Wakabayashi et al., 2006) to measure the autistic

13 tendencies of individuals. This scale is a self-administered questionnaire originally developed to measure

14 the degree of autistic traits of individuals with normal intelligence (Baron-Cohen et al., 2001b). This

15 questionnaire consists of 50 questions. Each question is rated on four choices: definitely agree (1),

16 slightly agree (2), slightly disagree (3), and definitely disagree (4). When the participant recorded

17 abnormal or autistic-like behavior as either definitely or slightly agree/disagree, 1 point was scored for the

18 item. We used only total scores for the analysis. Higher scores indicate higher autistic tendency. 
1 MRI acquisition

DTI data were acquired using single-shot spin-echo echoplanar sequences and structural MRI data

3 with 3-dimensional magnetization-prepared rapid gradient echo (3DMPRAGE) sequences, on a 3.0-T

4 MRI unit (Trio; Siemens, Erlangen, Germany) with a 40-mT/m gradient and a receiver-only eight-channel

5 phased-array head coil. The parameters for diffusion-weighted data were as follows: $\mathrm{TE}=96 \mathrm{~ms}$, TR =

$610,500 \mathrm{~ms}, 96 \times 96$ matrices, FOV $=192 \times 192 \mathrm{~mm}, 70$ contiguous axial slices of $2.0 \mathrm{~mm}$ thickness, 81

7 non-collinear axis motion probing gradient, $b=1,500 \mathrm{~s} / \mathrm{mm}^{2}$. The $b=0$ images were scanned preceding

8 every nine diffusion-weighted images, thus consisting of 90 volumes in total. Parameters for the

9 3D-MPRAGE imaging were as follows: $\mathrm{TE}=4.38 \mathrm{~ms}$; $\mathrm{TR}=2000 \mathrm{~ms}$, inversion time $=990 \mathrm{~ms}, 240 \times$

10256 matrices, FOV $=225 \times 240 \mathrm{~mm}$, resolution $=0.9375 \times 0.9375 \times 1.0 \mathrm{~mm}$, and 208 total axial sections

11 without intersection gaps.

12

13 Data preprocessing

All DTI data processing was performed using programs in the FMRIB Software Library (FSL)

15 version 4.1 .9 (http://www.fmrib.ox.ac.uk/fsl). Source data were corrected for eddy currents and head

16 motion by registering all data to the first $b=0$ image, with affine transformation. The FA maps were

17 calculated using the DTIFIT program implemented in FSL. For tractography, probability distributions on

182 fiber directions were modeled at each voxel using FSL's BedpostX program (Behrens et al., 2007),

19 based on a multifiber diffusion model. The 3D-MPRAGE images were preprocessed using the FreeSurfer 
1 software package version 5.0.0 (http://surfer.nmr.mgh .harvard.edu). In brief, the processing stream

2 included a Talairach transformation of each subject's native brain, removal of nonbrain tissue, volumetric

3 subcortical labeling, and surface-based segmentation of gray matter (GM)/white matter (WM) tissue. In

4 the automatic segmentation procedure, each voxel in the normalized brain volume was assigned a label

5 based on an atlas containing probabilistic information about the location of structures, such as the

6 thalamus, caudate, pallidum, putamen, accumbens area, hippocampus, amygdala, cerebral white matter,

7 cerebral cortex, brain stem, and non-brain regions. The cerebral cortex was parcellated into gyrus-based

8 regions of interest, which were used as seeds and targets for the following tractography.

10 Voxelwise DTI data analysis

11 TBSS version 1.2 was used for voxelwise statistical analysis. First, FA data of all subjects were

12 normalized into a common space using the nonlinear registration tool FNIRT; normalized FA images were

13 averaged to create a mean FA image, which was then thinned to create a skeleton taking only the centers

14 of white matter tracts. This skeleton was thresholded at FA of 0.2. Voxel values of each subject's

15 normalized FA map were projected onto the skeleton by searching the local maxima along the

16 perpendicular direction from the skeleton. Voxelwise permutation-based nonparametric inference

17 (Nichols and Holmes, 2002) was performed on this skeletonized FA data using FSL Randomize ver. 2.9.

18 We performed multiple regression analysis with AQ as covariate of interest and age and gender as

19 nuisance covariates. Both positive and negative correlations with AQ were tested, with 10,000 
1 permutations. The statistical threshold was set at $\mathrm{P}<0.05$. Multiple comparisons were corrected using

2 threshold-free cluster enhancement (TFCE) (Smith and Nichols, 2009). TFCE does not need an arbitrary

3 clusterforming threshold while preserving the sensitivity benefits of clusterwise correction. We identified

4 the location of the clusters using the Johns Hopkins University DTI-based White Matter Atlas

5 (http://cmrm.med.jhmi.edu) and checked potential fiber tracts that may include the clusters as follows.

7 Tractography

We used FSL's ProbtrackX program for depicting those fiber tracts. The seeds and targets of

9 tractography were selected from cortical parcellation created by FreeSurfer (Figure 1A). Exclusion masks

10 were also created from FreeSurfer parcellation to exclude anatomically invalid fibers. These masks were

11 transformed from each subject's 3D-MPRAGE space to the diffusion space by applying the rigid-body

12 transformation matrix, which was calculated by FSL's FLIRT program. Probabilistic tractography was

13 performed in the diffusion space (Figure 1B), and streamline samples were traced through the

14 probabilistic distributions of fiber direction, with 5,000 iterations per seed voxel (curvature thresholds =

15 0.5). Each tract was created in the 3D-MPRAGE space (Figure 1C) and thresholded to exclude voxels in

16 which the streamline sample count corresponded to the lower $25 \%$ of the outer-tail of the histogram, to

17 eliminate extraneous tracking results. The thresholded tracts were transformed back into diffusion spaces

18 (Figure 1D) and overlaid on the significant clusters of TBSS that were transformed into individual

19 diffusion spaces (Figure 1E,F). 
2 Identification of tracts

We tracked AF, ILF and IFOF. For AF, we chose the banks of the superior temporal sulcus as seed

4 and the middle frontal gyrus as target for tractography from parcellation of FreeSurfer. ILF was traced

5 from the temporal lobe to the extrastriatal region. For this analysis, we used the temporal pole, superior

6 temporal gyrus, middle temporal gyrus, inferior temporal gyrus and fusiform gyrus as temporal lobe from

7 parcellation of FreeSurfer. We also used the lateral occipital gyrus, cuneus and lingual gyrus as

8 extrastriatal regions in a similar way. Another tractography isolated IFOF by tracking from the

9 orbitofrontal area to the occipital lobe. We used lateral orbitofrontal, medial orbitofrontal and parsorbitalis

10 as the orbitofrontal area and lateral occipital, cuneus, pericalcarine and lingual gyrus as the occipital area

11 in a similar way.

12

13 Investigation of TBSS cluster

We tried to identify the fiber tracts upon which the significant cluster shown in the TBSS analysis

15 was located. For this purpose we calculated the extent of overlap between the cluster and each fiber tract.

16 Here we call each cluster transformed in diffusion space as the Cluster of Interest (Figure 1E,H), and the

17 overlap between a fiber tract and the Cluster of Interest as the Overlap (Figure 1G,I). We calculated the

18 Rate of Overlap as the volume of the Overlap divided by that of the Cluster of Interest. 


\section{Results}

The demographic data and AQ score are shown in Table.1. There was no gender difference in age

4 and AQ score. AQ was correlated with age. Although two participants had higher AQ score than the

5 cut-off level, we confirmed that they revealed no impairments in social interaction and communication in

6 a detailed interview by experienced psychiatrists.

TBSS revealed 5 clusters with a significantly negative correlation between FA and AQ (Table 2).

8 No clusters showed a significantly positive correlation. We did not find any significant correlation

9 between AQ and any of the other DTI parameters, such as mean, axial and radial diffusivity. According to

10 the JHU white matter atlas, the largest cluster (shown in light blue in Figure 2) appeared to fornix (Fx),

11 inferior longitudinal fasciculus (ILF), inferior fronto-occipital fasciculus (IFOF), posterior limb of

12 internal capsule (PLIC) and corona radiata. The second cluster (red in Figure 2) included the corona

13 radiata and corpus callosum (CC). The third cluster (green) included AF. The fourth (yellow) and fifth

14 (purple) clusters included the anterior thalamic radiation (Figure 2). The average FA value of each cluster

15 for each subject was extracted to confirm the correlations between FA and AQ score. The correlation

16 coefficients were significant with $\mathrm{r}=-.402,-.432,-.395,-.338$, and -.285 (each $\mathrm{P}<.05$ ), respectively. We

17 also conducted TBSS analysis with each subscale of AQ. We found no significant correlation between FA

18 and each subscale. 
1 largest cluster covered the white matter near pSTS. When shown in 3-dimensional (3D) view, it was

2 found that it could be divided into 4 subdivisions: 1) posterior limb of internal capsule and corona radiata,

3 2) fornix part, 3) occipital part, and 4) white matter close to pSTS (Figure 3). We picked the subcluster

4 adjacent to pSTS out from the mean FA image (standard space) manually and found that it had 413 voxels

5 and occupied the temporal stem from $y=-18$ to -41 and from $x=-28$ to -41 in Montreal Neurological

6 Institute coordinates. We transformed it into individual diffusion space and calculated the Rate of Overlap

7 in each of the fibers of IFOF, ILF and AF. The Rates of Overlap were $0.70( \pm 0.16)$ for the IFOF, 0.27

$8 \quad( \pm 0.17)$ for ILF and $0.04( \pm 0.06)$ for AF (Figure 4).

10 Discussion

11 We found that autistic traits in healthy adults were significantly correlated with the FA values of

12 white matter in several regions such as IFOF/ILF, PLIC/corona radiata, fornix, corpus callosum (CC), AF

13 and anterior thalamic radiation (ATR). In the region of pSTS, a key brain area in the pathophysiology of

14 ASD, we found altered white matter integrity in IFOF.

15 The fibers in which the current TBSS revealed FA negatively correlated with AQ were similar to

16 those where previous TBSS was found with reduced FA in ASD compared with controls (Jou et al., 2011b,

17 Shukla et al., 2011a). The locations of clusters were similar to those of previous studies reporting

18 IFOF/ILF, CC, AF and ATR. It was expected that no fiber showed a positive correlation in FA value with

19 AQ (Shukla et al., 2011b, Vissers et al., 2012). Similar white matter regions were, though to a lesser 
1 extent, also reported to be aberrant in unaffected siblings of children with ASD (Barnea-Goralyet al.,

2 2010). Thus, combined with these previous studies, our results indicate the existence of neural

3 underpinnings of autistic traits, which have ASD at one end and autistic tendency among healthy at on the

4 other.

These fiber tracts are known to be related to cognitive features of ASD. CC, for example, is related

6 to cognitive and social function (Paul et al., 2007). In addition, people with autism have altered white

7 matter integrity in CC (Alexander et al., 2007, Thomas et al., 2011). Our finding of the correlation

8 between FA value in CC and AQ are in line with these previous findings. We also found a negative

9 correlation between integrity in AF and AQ. AF is an important fiber for phonological processing (Duffau,

10 2012, Yeatman et al., 2011), which is impaired in children with ASD and their relatives (Wilson et al.,

11 2013). Our findings in this fiber were consistent with previous studies that showed altered integrity of AF

12 in ASD (Fletcher et al., 2010). Furthermore, we found a significant negative correlation between AQ and

13 FA in anterior thalamic radiation (ATR). Similar patterns of reduced FA of ATR were found in boys with

14 ASD (Cheon et al., 2011).

Analysis using tractography identified that the area with a negative correlation to AQ score was in

16 IFOF adjacent to the pSTS region. This area was detected as the cluster that seemed to continue from AF.

17 However, after confirming the fiber orientation of each individual with actual tractography, it was clear

18 that the parietal part and temporal part of AF were not present simultaneously in a sagittal plane because

19 AF runs backward from the frontal lobe and changes its course downward as well as laterally (Figure 4). 
1 According to the atlas, the cluster in pSTS appeared to be on IFOF or ILF, but it was difficult to specify

2 the individual fibers. By using tractography, we were able to determine the cluster on IFOF but not on

3 ILF.

4 IFOF seemed to be an important fiber for connecting the frontal lobe with other lobes, although

5 some researchers have insisted that IFOF did not exist (Schmahmann and Pandya, 2007). They claimed

6 that the associated fiber, which we call IFOF, did not exist in macaque monkey and that the fiber in the

7 external capsule was a projection fiber. However, researchers of postmortem human brain reported that

8 there is a fiber that runs from the frontal lobe to the occipital lobe via the external capsule, calling it IFOF

9 (Martino et al., 2010). DTI studies have also shown IFOF repeatedly (Catani et al., 2002, Wakana et al.,

10 2004), suggesting that it was peculiar to human beings (Thiebaut de Schotten et al., 2012). Research on

11 the function of IFOF with intraoperative electrical stimulation has revealed its role in semantic processing

12 (Duffau et al., 2005). A study of brain injury also showed that IFOF was related to semantic processing

13 (Han et al., 2013), which has been reported to be impaired in people with ASD (Boucher, 2012).

14 Furthermore, a study of brain injury indicated that IFOF was associated with perception of facial

15 expression (Philippi et al., 2009). The processing of facial expression is aberrant in people with ASD

16 (Losh et al., 2009, Weng et al., 2011). Thus, variation of fiber integrity of IFOF might lead to autistic

17 features, as the fiber plays a key role in processing social information.

We experienced several limitations in this study. First of all, we did not investigate the brains of

19 individuals with ASD. Further study needs to include both subjects with and without ASD. On the other 
1 hand, some subjects with ASD have mental retardation, which might affect the results. The current study

2 can remove this confounding factor because all of our subjects had normal IQ. Second, we could not

3 distinguish IFOF from extreme capsule (EmC). The part of EmC, not IFOF, might have an alteration of

$4 \quad$ FA associated with AQ. Finally, it was not revealed which cortices the altered white matter actually

5 connects, although we found that the pSTS parts of IFOF have reductions of FA values.

6 In conclusion, we showed that typically developed individuals with high autistic traits tend to show

7 lower FA in several brain areas including pSTS. Furthermore, by combining TBSS and tractography, we

8 were able to reveal that the white matter associated with autistic trait was mainly on IFOF in the pSTS

9 area. Our findings suggest that reduced white matter integrity in pSTS in ASD might stem from

10 disruption of IFOF. This result from healthy adults could contribute to a better understanding of the whole

11 pathological mechanism underlying ASD. 


\section{References}

2 Alexander AL, Lee JE, Lazar M, Boudos R, DuBray MB, Oakes TR, et al. Diffusion tensor imaging of 3 the corpus callosum in Autism. Neuroimage. 2007;34:61-73.

4 Allely CS, Gillberg C, Wilson P. Neurobiological Abnormalities in the First Few Years of Life in 5 Individuals Later Diagnosed with Autism Spectrum Disorder: A Review of Recent Data. Behavioural 6 Neurology. 2014.

7 American Psychiatric Association. The Diagnostic and Statistical Manual of Mental Disorders. $5^{\text {th }}$ ed. 8 Arlington: American Psychiatric Association; 2013.

9 Barnea-Goraly N, Kwon H, Menon V, Eliez S, Lotspeich L, Reiss AL. White matter structure in autism: 10 Preliminary evidence from diffusion tensor imaging. Biol Psychiat. 2004;55:54s-s.

11 Barnea-Goraly N, Lotspeich LJ, Reiss AL. Similar White Matter Aberrations in Children With Autism 12 and Their Unaffected Siblings A Diffusion Tensor Imaging Study Using Tract-Based Spatial Statistics. 13 Arch Gen Psychiat. 2010;67:1052-60.

14 Baron-Cohen S, Wheelwright S, Hill J, Raste Y, Plumb I. The "Reading the Mind in the Eyes" test revised 15 version: A study with normal adults, and adults with Asperger syndrome or high-functioning autism.

16 Journal of Child Psychology and Psychiatry and Allied Disciplines. 2001a;42:241-51.

17 Baron-Cohen S, Wheelwright S, Skinner R, Martin J, Clubley E. The Autism-Spectrum Quotient (AQ):

18 Evidence from Asperger syndrome/high-functioning autism, males and females, scientists and 19 mathematicians. J Autism Dev Disord. 2001b;31:5-17.

20 Behrens TEJ, Berg HJ, Jbabdi S, Rushworth MFS, Woolrich MW. Probabilistic diffusion tractography 21 with multiple fibre orientations: What can we gain? Neuroimage. 2007;34:144-55.

22 Bloemen OJN, Deeley Q, Sundram F, Daly EM, Barker GJ, Jones DK, et al. White Matter Integrity in 23 Asperger Syndrome: A Preliminary Diffusion Tensor Magnetic Resonance Imaging Study in Adults. 24 Autism Res. 2010;3:203-13.

25 Boucher J. Research Review: Structural language in autistic spectrum disorder - characteristics and 26 causes. J Child Psychol Psyc. 2012;53:219-33.

27 Catani M, de Schotten MT. Atlas of human brain connections: Oxford University Press; 2012.

28 Catani M, Howard RJ, Pajevic S, Jones DK. Virtual in vivo interactive dissection of white matter fasciculi 29 in the human brain. Neuroimage. 2002;17:77-94.

30 Cauda F, Geda E, Sacco K, D'Agata F, Duca S, Geminiani G, et al. Grey matter abnormality in autism 31 spectrum disorder: an activation likelihood estimation meta-analysis study. Journal of Neurology,

32 Neurosurgery \& Psychiatry. 2011;82:1304-13.

33 Cheon K-A, Kim Y-S, Oh S-H, Park S-Y, Yoon H-W, Herrington J, et al. Involvement of the anterior 34 thalamic radiation in boys with high functioning autism spectrum disorders: a diffusion tensor imaging 35 study. Brain research. 2011;1417:77-86.

36 Constantino JN, Todd RD. Autistic traits in the general population - A twin study. Arch Gen Psychiat. $37 \quad 2003 ; 60: 524-30$. 
1 Courchesne E. Brainstem, cerebellar and limbic neuroanatomical abnormalities in autism. Current

2 opinion in neurobiology. 1997;7:269-78.

3 Duffau H. The "frontal syndrome" revisited: lessons from electrostimulation mapping studies. Cortex. 4 2012;48:120-31.

5 Duffau H, Gatignol P, Mandonnet E, Peruzzi P, Tzourio-Mazoyer N, Capelle L. New insights into the 6 anatomo-functional connectivity of the semantic system: a study using cortico-subcortical 7 electrostimulations. Brain. 2005;128:797-810.

8 Fletcher PT, Whitaker RT, Tao R, DuBray MB, Froehlich A, Ravichandran C, et al. Microstructural 9 connectivity of the arcuate fasciculus in adolescents with high-functioning autism. Neuroimage.

$10 \quad 2010 ; 51: 1117-25$.

11 Groen WB, Buitelaar JK, van der Gaag RJ, Zwiers MP. Pervasive microstructural abnormalities in 12 autism: a DTI study. J Psychiatr Neurosci. 2011;36:32-40.

13 Han ZZ, Ma YJ, Gong GL, He Y, Caramazza A, Bi YC. White matter structural connectivity underlying 14 semantic processing: evidence from brain damaged patients. Brain. 2013;136:2952-65.

15 Jou RJ, Jackowski AP, Papademetris X, Rajeevan N, Staib LH, Volkmar FR. Diffusion tensor imaging in 16 autism spectrum disorders: preliminary evidence of abnormal neural connectivity. Aust Nz J Psychiat. 17 2011a;45:153-62.

18 Jou RJ, Mateljevic N, Kaiser MD, Sugrue DR, Volkmar FR, Pelphrey KA. Structural Neural Phenotype of 19 Autism: Preliminary Evidence from a Diffusion Tensor Imaging Study Using Tract-Based Spatial 20 Statistics. Am J Neuroradiol. 2011b;32:1607-13.

21 Just MA, Keller TA, Malave VL, Kana RK, Varma S. Autism as a neural systems disorder: A theory of 22 frontal-posterior underconnectivity. Neurosci Biobehav R. 2012;36:1292-313.

23 Kana RK, Libero LE, Moore MS. Disrupted cortical connectivity theory as an explanatory model for 24 autism spectrum disorders. Phys Life Rev. 2011;8:410-37.

25 Kumar A, Sundaram SK, Sivaswamy L, Behen ME, Makki MI, Ager J, et al. Alterations in Frontal Lobe 26 Tracts and Corpus Callosum in Young Children with Autism Spectrum Disorder. Cereb Cortex.

27 2010;20:2103-13.

28 Lahnakoski JM, Glerean E, Salmi J, Jaaskelainen I, Sams M, Hari R, et al. Naturalistic fMRI mapping 29 reveals superior temporal sulcus as the hub for the distributed brain network for social perception. Front 30 Hum Neurosci. 2012;6.

31 Losh M, Adolphs R, Poe MD, Couture S, Penn D, Baranek GT, et al. Neuropsychological Profile of 32 Autism and the Broad Autism Phenotype. Arch Gen Psychiat. 2009;66:518-26.

33 Martino J, Brogna C, Robles SG, Vergani F, Duffau H. Anatomic dissection of the inferior fronto-occipital 34 fasciculus revisited in the lights of brain stimulation data. Cortex. 2010;46:691-9.

35 Nichols TE, Holmes AP. Nonparametric permutation tests for functional neuroimaging: A primer with 36 examples. Hum Brain Mapp. 2002;15:1-25.

37 Nummenmaa L, Engell AD, von dem Hagen E, Henson RNA, Calder AJ. Autism spectrum traits predict 38 the neural response to eye gaze in typical individuals. Neuroimage. 2012;59:3356-63. 
1 Paul LK, Brown WS, Adolphs R, Tyszka JM, Richards LJ, Mukherjee P, et al. Agenesis of the corpus

2 callosum: genetic, developmental and functional aspects of connectivity. Nature Reviews Neuroscience.

$3 \quad 2007 ; 8: 287-99$.

4 Pelphrey KA, Shultz S, Hudac CM, Wyk BCV. Research Review: Constraining heterogeneity: the social 5 brain and its development in autism spectrum disorder. J Child Psychol Psyc. 2011;52:631-44.

6 Philippi CL, Mehta S, Grabowski T, Adolphs R, Rudrauf D. Damage to Association Fiber Tracts Impairs 7 Recognition of the Facial Expression of Emotion. J Neurosci. 2009;29:15089-99.

8 Robinson EB, Munir K, Munafo MR, Hughes M, McCormick MC, Koenen KC. Stability of Autistic

9 Traits in the General Population: Further Evidence for a Continuum of Impairment. J Am Acad Child Psy. $10 \quad 2011 ; 50: 376-84$.

11 Schmahmann JD, Pandya DN. The complex history of the fronto-occipital fasciculus. J Hist Neurosci. $12 \quad 2007 ; 16: 362-77$.

13 Shukla DK, Keehn B, Muller RA. Tract-specific analyses of diffusion tensor imaging show widespread 14 white matter compromise in autism spectrum disorder. J Child Psychol Psyc. 2011a;52:286-95.

15 Shukla DK, Keehn B, Smylie DM, Muller RA. Microstructural abnormalities of short-distance white 16 matter tracts in autism spectrum disorder. Neuropsychologia. 2011b;49:1378-82.

17 Smith SM, Jenkinson M, Johansen-Berg H, Rueckert D, Nichols TE, Mackay CE, et al. Tract-based 18 spatial statistics: Voxelwise analysis of multi-subject diffusion data. Neuroimage. 2006;31:1487-505.

19 Smith SM, Nichols TE. Threshold-free cluster enhancement: Addressing problems of smoothing, 20 threshold dependence and localisation in cluster inference. Neuroimage. 2009;44:83-98.

21 Stanfield AC, McIntosh AM, Spencer MD, Philip R, Gaur S, Lawrie SM. Towards a neuroanatomy of 22 autism: A systematic review and meta-analysis of structural magnetic resonance imaging studies.

23 European Psychiatry. 2008;23:289-99.

24 Thiebaut de Schotten M, Dell'Acqua F, Valabregue R, Catani M. Monkey to human comparative anatomy 25 of the frontal lobe association tracts. Cortex. 2012;48:82-96.

26 Thomas C, Humphreys K, Jung K-J, Minshew N, Behrmann M. The anatomy of the callosal and 27 visual-association pathways in high-functioning autism: a DTI tractography study. Cortex. $28 \quad 2011 ; 47: 863-73$.

29 Via E, Radua J, Cardoner N, Happe F, Mataix-Cols D. Meta-analysis of Gray Matter Abnormalities in 30 Autism Spectrum Disorder Should Asperger Disorder Be Subsumed Under a Broader Umbrella of 31 Autistic Spectrum Disorder? Arch Gen Psychiat. 2011;68:409-18.

32 Vissers ME, Cohen MX, Geurts HM. Brain connectivity and high functioning autism: A promising path 33 of research that needs refined models, methodological convergence, and stronger behavioral links.

34 Neurosci Biobehav R. 2012;36:604-25.

35 Wakabayashi A, Baron-Cohen S, Wheelwright S, Tojo Y. The Autism-Spectrum Quotient (AQ) in Japan: 36 A cross-cultural comparison. J Autism Dev Disord. 2006;36:263-70.

37 Wakana S, Jiang HY, Nagae-Poetscher LM, van Zijl PCM, Mori S. Fiber tract-based atlas of human white 38 
1 Walker L, Gozzi M, Lenroot R, Thurm A, Behseta B, Swedo S, et al. Diffusion Tensor Imaging in Young

2 Children with Autism: Biological Effects and Potential Confounds. Biol Psychiat. 2012;72:1043-51.

3 Wallace GL, Shaw P, Lee NR, Clasen LS, Raznahan A, Lenroot RK, et al. Distinct cortical correlates of

4 autistic versus antisocial traits in a longitudinal sample of typically developing youth. The Journal of

5 neuroscience : the official journal of the Society for Neuroscience. 2012;32:4856-60.

6 Weng SJ, Carrasco M, Swartz JR, Wiggins JL, Kurapati N, Liberzon I, et al. Neural activation to

7 emotional faces in adolescents with autism spectrum disorders. J Child Psychol Psyc. 2011;52:296-305.

8 Wilson LB, Tregellas JR, Slason E, Pasko BE, Hepburn S, Rojas DC. Phonological processing in

9 first - degree relatives of individuals with autism: An fMRI study. Human brain mapping.

$10 \quad 2013 ; 34: 1447-63$.

11 Wing L. The continuum of autistic characteristics. In: Shopler E, Mesibov GB, editors. Diagnosis and

12 assessment in autism. New York: Springer; 1988. p. 91-110

13 Wing L. The autistic spectrum. Lancet. 1997;350:1761-6.

14 Wolff JJ, Gu HB, Gerig G, Elison JT, Styner M, Gouttard S, et al. Differences in White Matter Fiber Tract

15 Development Present From 6 to 24 Months in Infants With Autism. Am J Psychiat. 2012;169:589-600.

16 Yeatman JD, Dougherty RF, Rykhlevskaia E, Sherbondy AJ, Deutsch GK, Wandell BA, et al. Anatomical

17 properties of the arcuate fasciculus predict phonological and reading skills in children. Journal of

18 cognitive neuroscience. 2011;23:3304-17.

19 Zilbovicius M, Meresse I, Chabane N, Brunelle F, Samson Y, Boddaert N. Autism, the superior temporal

20 sulcus and social perception. Trends Neurosci. 2006;29:359-66. 


\section{$1 \quad$ Legends}

2 Figure 1

3 The scheme of tracking fibers and identifying locations of TBSS clusters.

4 A, A 3D-MPRAGE structural image of an individual brain with seeds obtained from FreeSurfer cortical 5 parcellation.

6 B, A B0 image. Probtrackx tracked fibers in diffusion space, using seeds defined in A.

7 C, A structural image with seeds and an identified fiber. Probtrackx produces the results in the same space 8 as seeds.

9 D, The fiber tract transferred into diffusion space.

10 E, A significant cluster from TBSS, transformed from MNI152 space into each subject's diffusion space.

11 F, The fiber tract overlapped with TBSS cluster.

12 G, The fiber tract and TBSS cluster in the sagittal plane. The overlapped region is colored in magenta.

$13 \mathrm{H}, \mathrm{A}$ TBSS cluster in the sagittal plane. We call this the Cluster of Interest.

14 I, A TBSS cluster with the overlapped part in the sagittal plane. We call magenta part as the Overlap.

15 TBSS, tract-based special statistics; MNI, Montreal Neurological Institute

16 
$1 \quad$ Figure 2

2 Images of all clusters in standardized brain with FA values negatively correlated with AQ. Colors change

3 by clusters. Copper-colored region is skeleton mask. Yellow lines in the left upper picture show the

4 position of each slice. Light blue region is the largest cluster, and red is the second largest. The $3^{\text {rd }}, 4^{\text {th }}$ and

$55^{\text {th }}$ clusters are green, purple and yellow, respectively. R: right, L: left, P: posterior, A: anterior, FA:

6 fractional anisotropy, AQ: autism spectrum quotient, 
$1 \quad$ Figure 3

2 Colored 3D images of the light blue cluster of interest from Figure 1 as seen on standardized brain. Green,

3 red, light blue and blue regions comprise this largest cluster correlated negatively with AQ in TBSS.

4 These 4 subclusters represent occipital part (green), fornix part (red), corona radiata part (light blue) and

5 blue colored region. The blue region was taken away from the whole cluster manually because it was

6 located adjacent to pSTS. Yellow bars are axes on MNI coordinates. A, Image viewed from right side.

7 Orange arrow shows C and light green arrow shows D. B, Image viewed from left side. Bigger clusters

8 than A can be seen because they are located in the left hemisphere. Purple arrow shows E. C, Partial

9 image viewed from posterior upper position. Green part is located far from light blue part. D, Partial

10 image viewed from left backward position. Green part has a gap with blue part. Light blue part also has a

11 gap with blue part. E, Partial image viewed from lower right position. Blue part is contiguous with red

12 part only by 1 voxel. R: right, L: left, P: posterior, A: anterior, 3D: 3-dimensional, AQ: autism spectrum

13 quotient, TBSS: tract-based spatial statistics, pSTS: posterior superior temporal sulcus, MNI: Montreal

14 Neurological Institute

15

16

17 
$1 \quad$ Figure 4

2 Images of clusters and fiber tracts found by TBSS and tractography. A, Sagittal slice showing cluster on

3 pSTS. B, Picture of individual's brain showing similar slice to A and projected on the results of

4 tractography. Cool colors (light blue and purple) show AF. Hot colors (red and white) show ILF. Yellow

5 band shows IFOF. Magenta color shows overlap between ILF and IFOF. Blue is ROI and green is 3rd

6 cluster. Green line shows coronal slices C and D. Yellow line shows E and F. C, Coronal slice on

7 standardized brain. D, Coronal slice on individual's FA image. Blue cluster is not on AF, as AF is more

8 lateral going downward. E, Another coronal slice. F, Another coronal slice on individual's FA image. Blue

9 cluster is on IFOF, not ILF. TBSS, tract-based special statistics; pSTS, posterior superior temporal sulcus;

10 AF, arcuate fasciculus; ILF, inferior longitudinal fasciculus; IFOF, inferior fronto-occipital fasciculus;

11 ROI, region of interest; R, right; L, left; P, posterior; A, anterior, FA: fractional anisotropy,

12 


\section{Highlights}

TBSS revealed that autistic traits are associated with white matter integrity.

Tractography identified the fiber tract in which FA was linked to autistic traits.

The fiber tract in pSTS region was the inferior fronto-occipital fasciculus. 
Figure 1

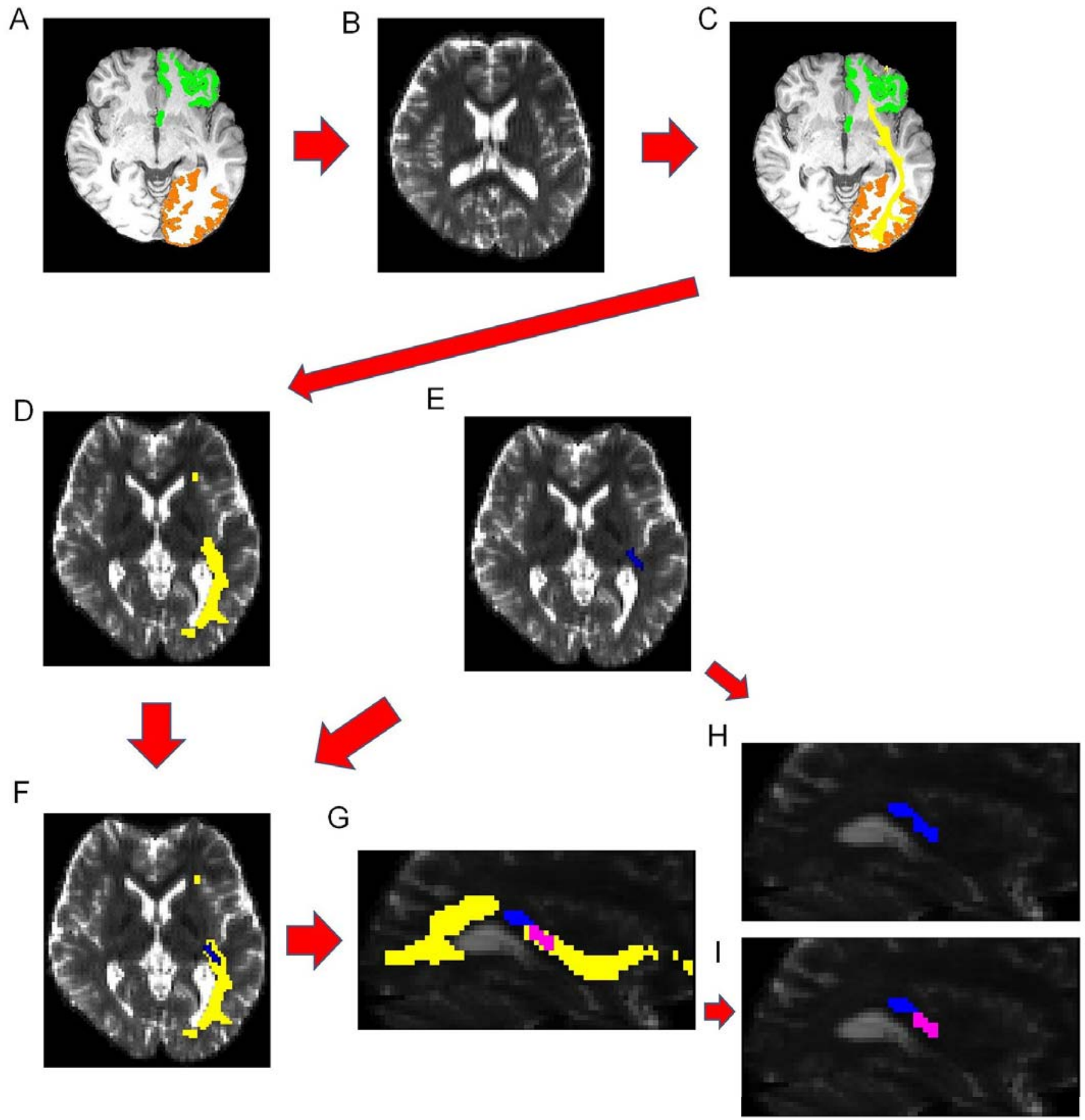


Figure 2
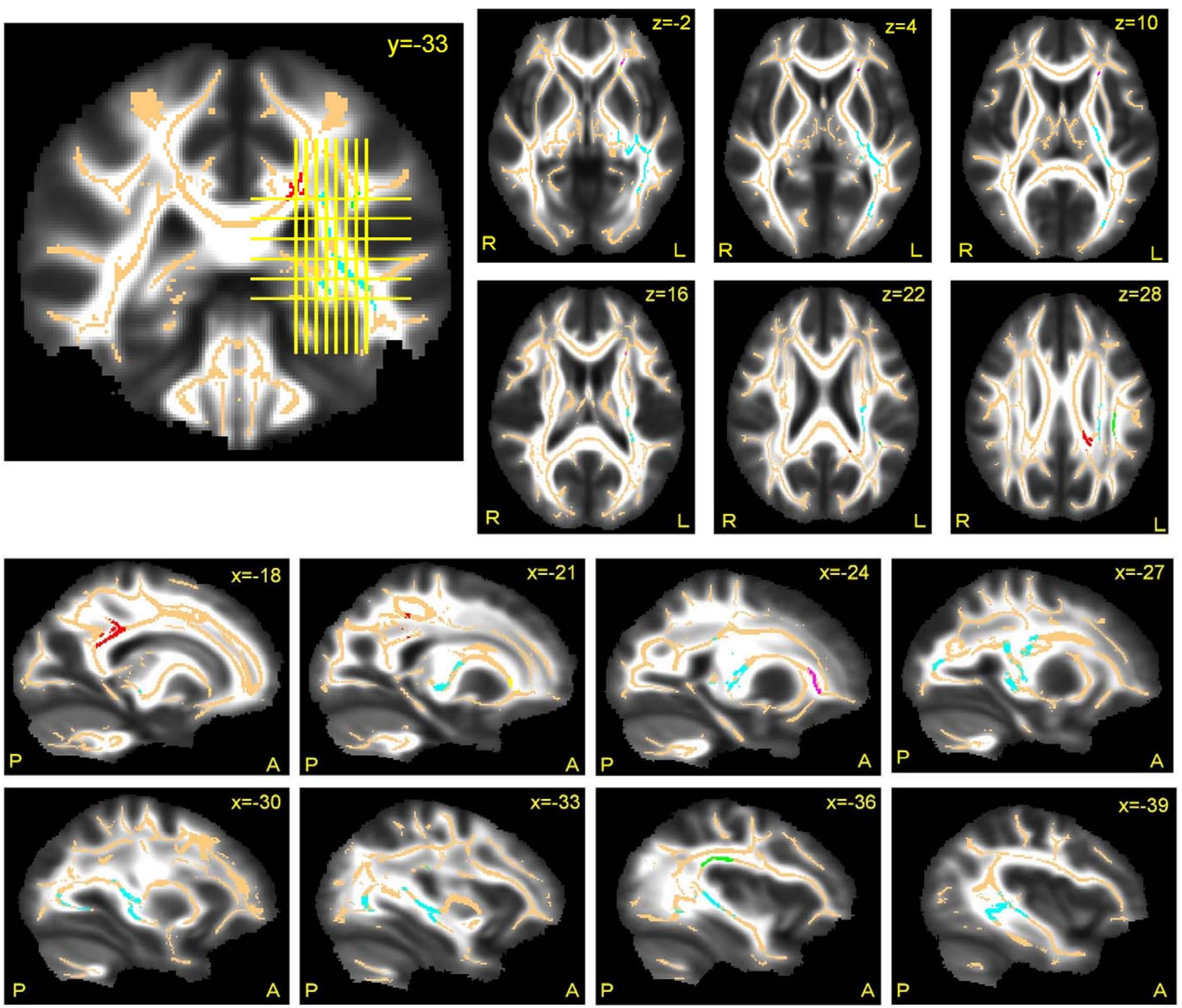
Figure 3
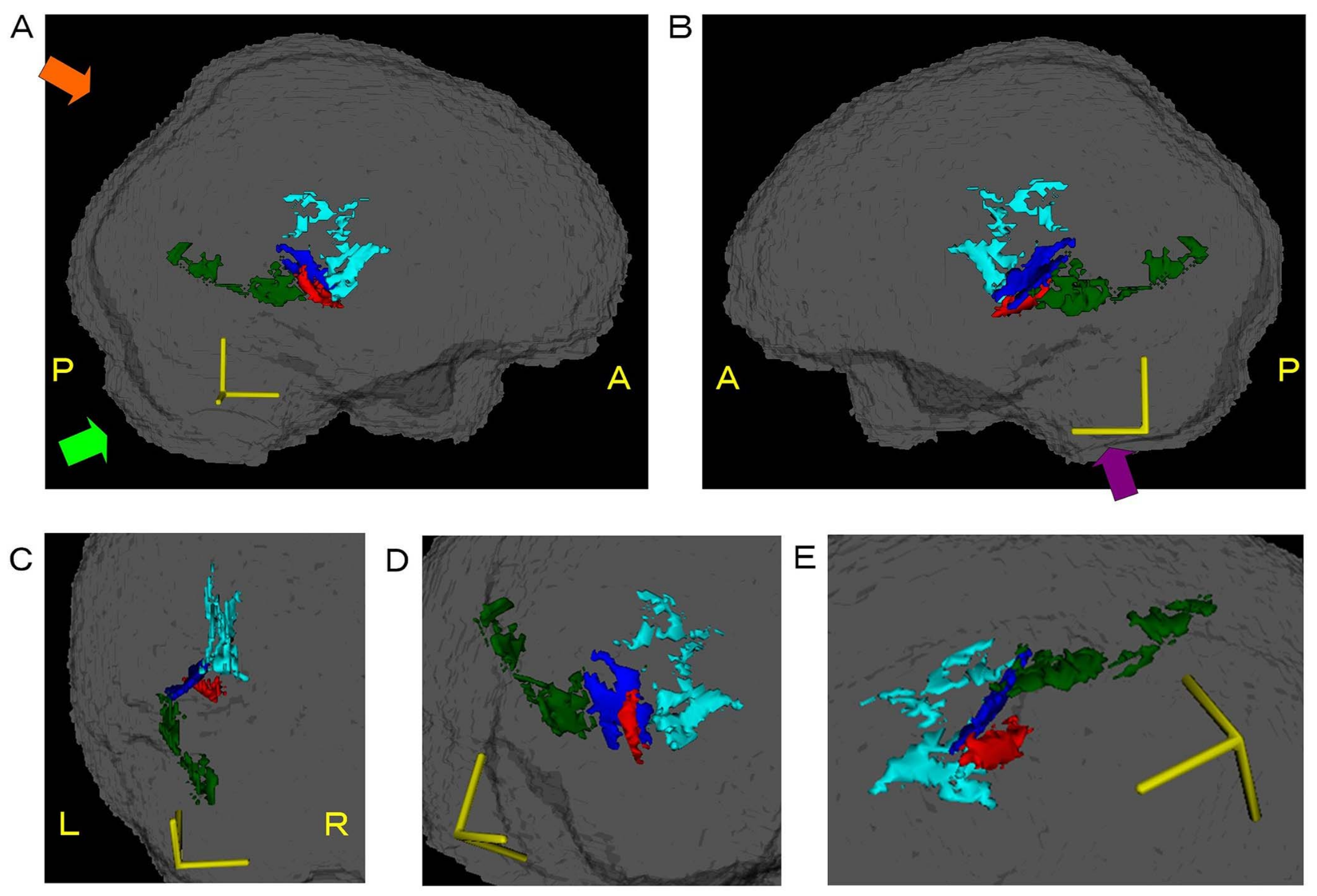
Figure 4
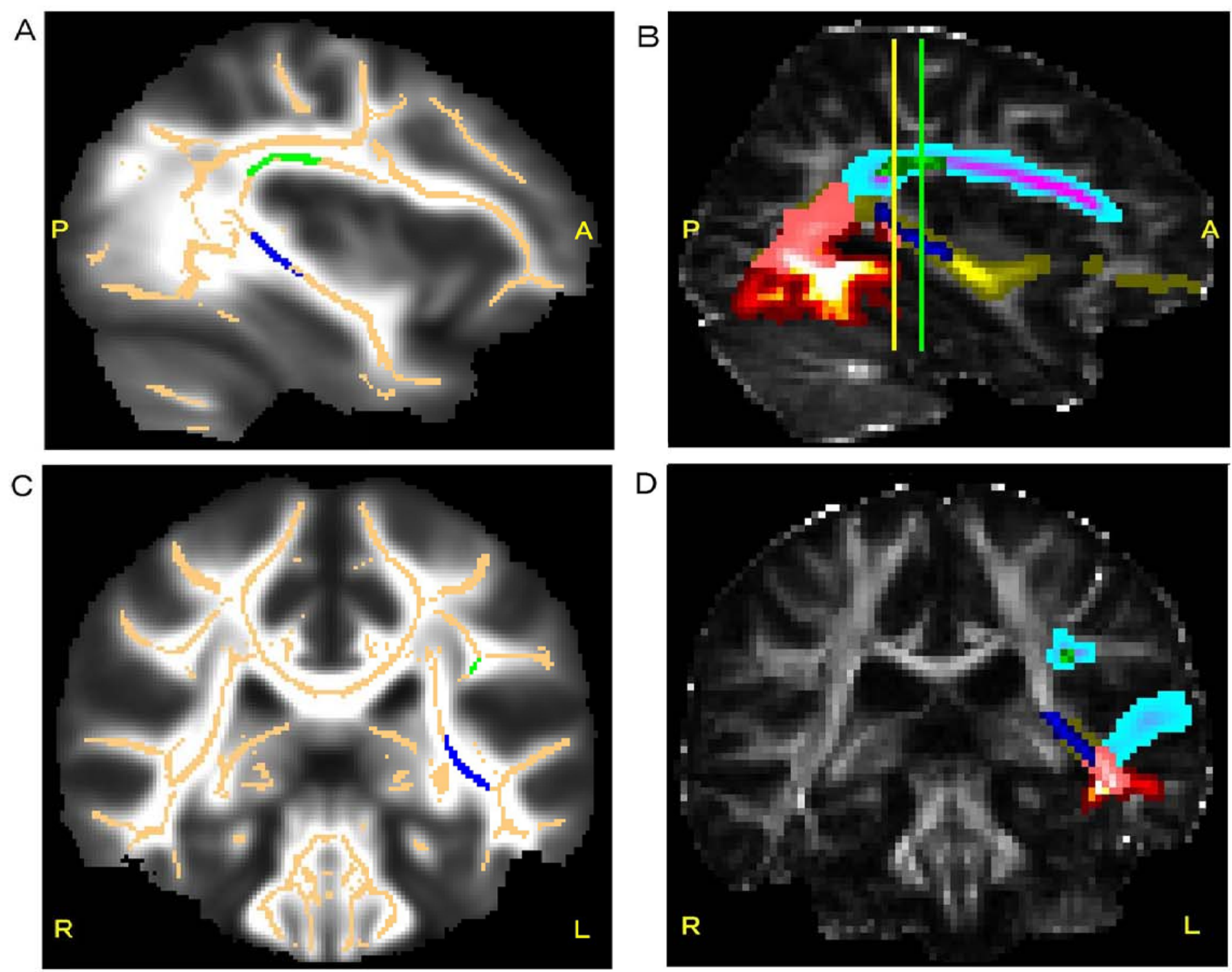

E

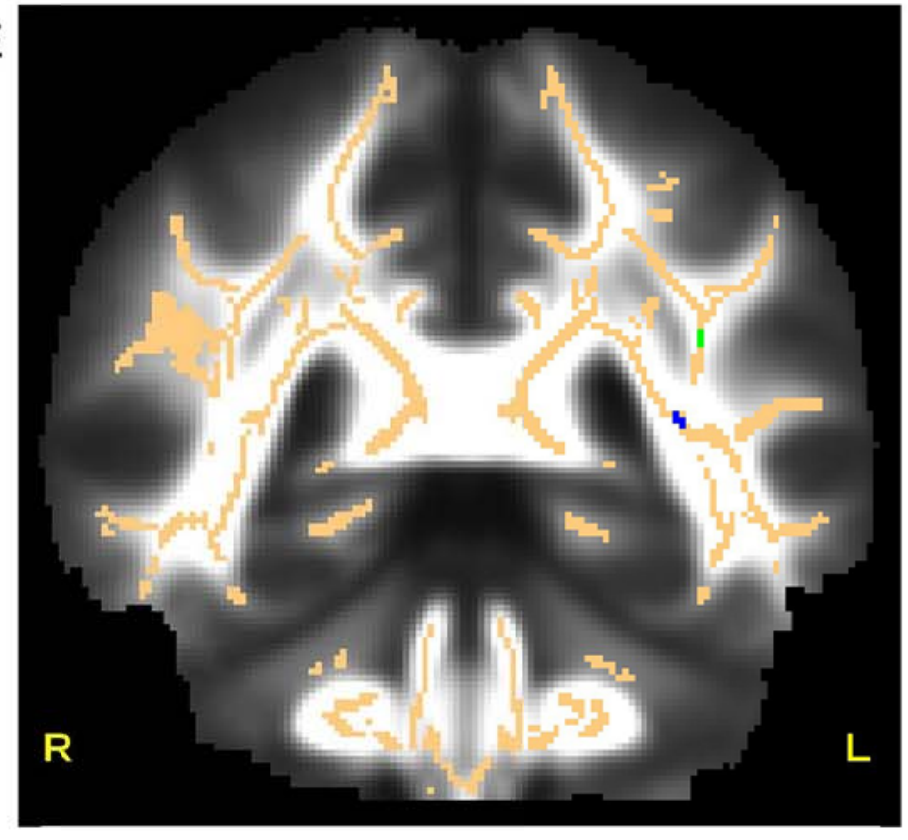

F

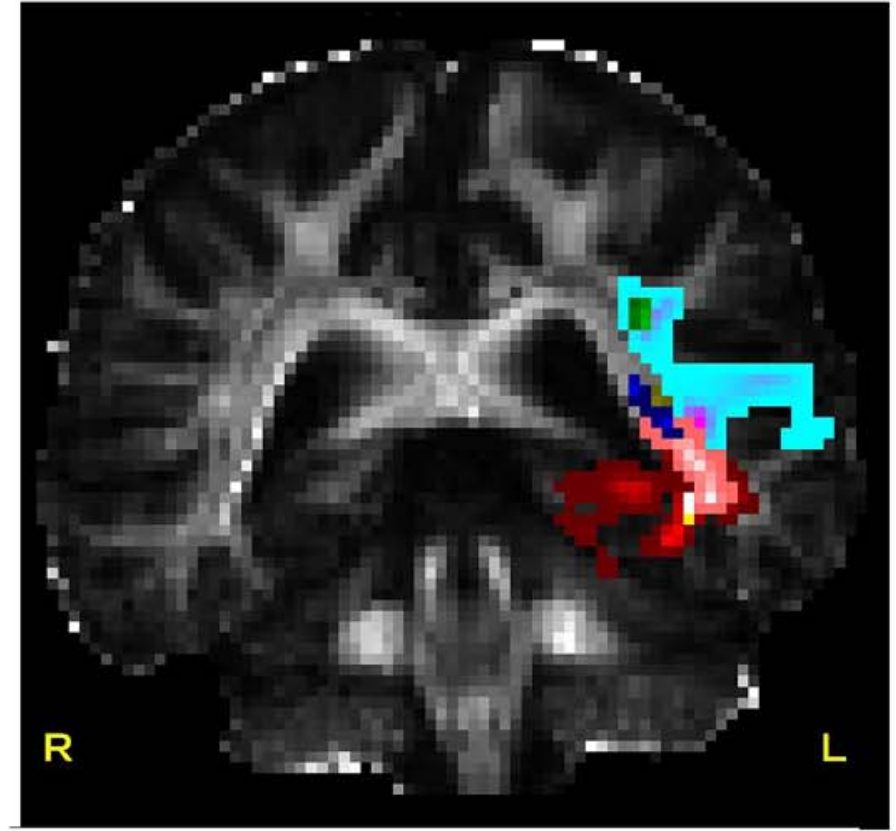


Table 1

Demographic data

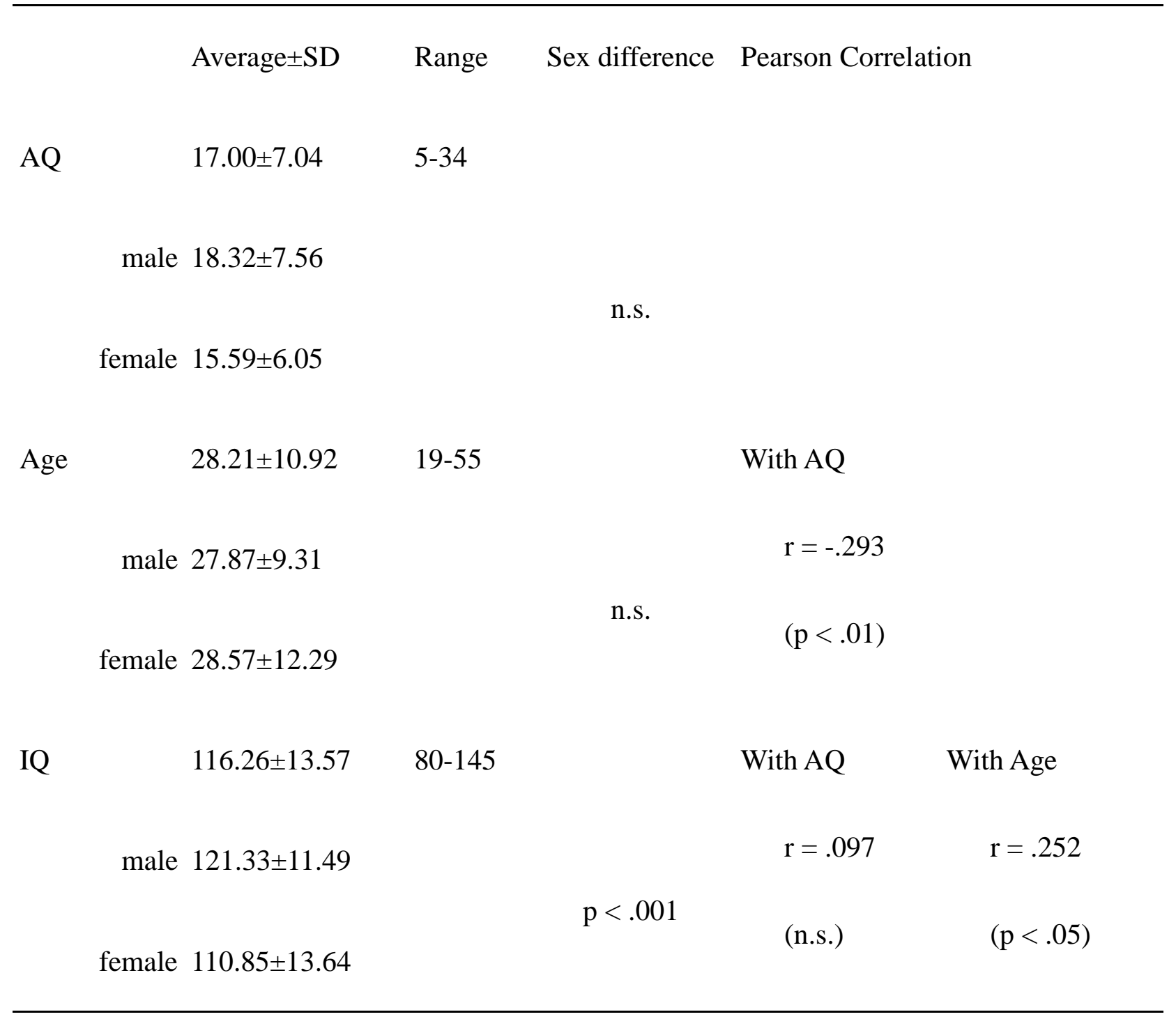

Distribution of AQ is similar to that expected. SD, standard deviation; AQ, autism spectrum quotient; IQ, intelligence quotient; n.s., not significant 
Table 2

Results of TBSS

Area or possible tract fibers

voxels

t value

Z MAX

X

y $\quad$ z

1) PLIC/corona radiata, IFOF, ILF, Fx

1708

4.02

$-40$

$-44$

$-3$

2) corona radiata, $\mathrm{CC}$

373

5.01

$-17 \quad-36$

28

3) arcuate fasciculus

128

4

$-35$

$-23$

26

4) anterior thalamic radiation

69

2.86

$-25$

$27 \quad 11$

5) anterior thalamic radiation

45

3.03

$-21$

23

$-1$

5 clusters were found to be negatively correlated with AQ. TBSS, tract-based spatial statistics; MAX, maximum; PLIC, posterior limb of internal capsule; IFOF, inferior fronto-occipital fasciculus; ILF, inferior longitudinal fasciculus; Fx, fornix; CC, corpus callosum; AQ, autism spectrum quotient 